\title{
Clinical Report on Pharmacological Prevention of Deep Vein Thrombosis after Radical Resection of Abdominal Tumors
}

\author{
Liang He ${ }^{*}$, Jiang Wang, Lin Nan, Bin Yan, Yongjiu Zhang \\ Department of General Surgery, Lanzhou Military District Hospital in Urumqi, Urumqi, China. \\ Email: *heliangfmmu@sina.com
}

Received May 24 ${ }^{\text {th }}$, 2013; revised June 20 $0^{\text {th }}$ 2013; accepted July $18^{\text {th }}, 2013$

Copyright (C 2014 Liang He et al. This is an open access article distributed under the Creative Commons Attribution License, which permits unrestricted use, distribution, and reproduction in any medium, provided the original work is properly cited. In accordance of the Creative Commons Attribution License all Copyrights (C) 2014 are reserved for SCIRP and the owner of the intellectual property Liang He et al. All Copyright (C) 2014 are guarded by law and by SCIRP as a guardian.

\begin{abstract}
Aim: The aim of this paper is to summarize the experience and results of deep vein thrombosis prevention after standardized abdominal tumor resection and lymph node dissection, and to investigate standardized treatment methods for postoperative thrombosis prevention. Methods: We performed a retrospective analysis of the clinical data for 548 patients who were given low molecular weight heparin, low molecular weight dextran, or IV salvia to prevent thrombosis development between January 2007 and April 2010 after standardized abdominal tumor resection and lymph node dissection. Patients were divided into the following three groups based on the treatment period and treatment schedule: group 1 included 163 patients who had been treated between January 2007 and March 2008 and received a 7-day course of daily low molecular weight dextran $(500 \mathrm{~mL})$ and salvia injection ( $0.2 \mathrm{~g})$ postoperatively; group 2 included 149 patients who had been treated between April 2008 and March 2009 and received a 7-day course of daily low molecular weight dextran $(500 \mathrm{~mL})$, salvia injection $(0.2 \mathrm{~g})$, and low molecular weight heparin $(40 \mathrm{mg})$ postoperatively; group 3 included 236 patients who had been treated between April 2009 and April 2010 and postoperatively received a 7-day course of daily low molecular weight dextran $(500 \mathrm{~mL})$ and salvia injection $(0.2 \mathrm{~g})$, and on postoperative day 3 were started on an additional 7-day course of daily low molecular weight heparin $(40 \mathrm{mg})$. Then, we performed comparative analysis of the treatment efficacy and concomitant symptoms. Results: In group 1, 64 of the 163 cases $(39.3 \%)$ were positive for D-dimer, and $15(9.2 \%)$ cases were positive for DVT based on Doppler imaging of the lower extremities. In group 2, 38 cases (25.5\%) were positive for D-dimer and 3 cases $(2.0 \%)$ for DVT. In group 3, 62 cases (26.3\%) were positive for D-dimer and 6 cases $(2.5 \%)$ for DVT. In general, the administration of low molecular weight heparin for anticoagulation in groups 2 and 3 led to significant reduction of thromboses when compared to group 1. However, altering the initiation day of low molecular weight heparin administration between groups 2 and 3 did not result in significant differences in the rate of thrombosis formation. Through clinical observation, early administration of low molecular weight heparin may increase adverse effects. Conclusion: We have demonstrated the superior efficacy of postoperative administration of low molecular weight dextran and IV salvia with additional subcutaneous injection of low molecular weight heparin on postoperative day 3 to prevent DVT development after radical resection of abdominal tumors.
\end{abstract}

\section{KEYWORDS}

Abdominal Tumor; Venous Thrombosis; Surgery

\section{Introduction}

Deep venous thrombosis (DVT) is a serious complication

\footnotetext{
*Corresponding author.
}

that occurs after major abdominal surgeries. Complications can vary, and severe cases can be fatal due to thrombotic embolism [1]. Existing data show an incidence rate for DVT of $10 \%$ to $63 \%$ after orthopedic and 
general surgery. In addition, postoperative patients with malignant tumors are also at high risk for DVT [2]. Treatment after the formation of thrombosis is often not ideal; therefore, active preventive treatment is critical. In January 2007, the gastrointestinal surgery center at our institution began standardized administration of low molecular weight dextran and IV salvia for patients with standardized resection of stomach, intestine, hepatobiliary, and pancreatic tumors and subsequent lymph node dissection. Starting in April 2008, we have additionally incorporated low molecular weight heparin. By comparing the three clinical stages of our study, we optimized the drug delivery duration and dosage.

\section{Materials and Methods}

\subsection{General Information}

Between January 2007 and April 2010, the gastrointestinal surgery center at our institution performed standardized surgical resections of stomach, intestine, hepatobiliary, and pancreatic tumors and lymph node dissection in 548 patients. The study population consisted of 412 males and 136 are females (male to female ratio $=3: 1$ ) with an age range from 30 to 92 years (average $=56.0 \pm$ 9.2 years). Radical tumor resections included gastric cancer (243 cases), rectal cancer (140 cases), liver cancer (87 cases), gallbladder cancer (26 cases), pancreatic and ampullary cancer (52 cases). All patients were in good preoperative condition and had no significantly abnormal liver function or blood coagulation and no history of DVT or long-term use of anticoagulation medication. During surgery, complete hemostasis was obtained and no significant perioperative bleeding or errhysis was noted. The study population was divided into three groups by based on timing of three pharmacological adjustments. The sex and age ratios between the three groups were comparable and not significantly different.

\subsection{Grouping and Drug Regimens}

Group 1: Tumor resection surgeries and lymph node dissection procedures were performed between January 2007 and March 2008 in 163 patients. A 7-day course of $500 \mathrm{ml}$ low molecular weight dextran and $0.2 \mathrm{~g}$ IV salvia was initiated immediately after surgery. Group 2: Surgeries were performed in 149 patients between April 2008 and March 2009 and a 7-day course of $500 \mathrm{ml}$ low molecular weight dextran, $0.2 \mathrm{~g}$ IV salvia, and $40 \mathrm{mg}$ low molecular weight heparin was initiated immediately after surgery. Group 3: Surgery was performed in 236 patients between April 2009 and April 2010 and a 7-day course of $500 \mathrm{ml}$ low molecular weight dextran and $0.2 \mathrm{~g} \mathrm{IV}$ salvia was administered immediately after the surgery, while a 7-day course of $40 \mathrm{mg}$ low molecular weight heparin was initiated three days after surgery. The medication dosages were adjusted in some patients based on body weight. In cases of concurrent splenectomy, the postoperative platelet count was monitored and, if the value reached more than twice the reference value, a dose of $75 \mathrm{mg}$ clopidogrel was administered orally while the original anticoagulant therapy was terminated. For well-defined thrombosis cases, standardized thrombolytic, anticoagulant, anti-platelet-adhesion therapies were administered. After successful thrombolysis, the patient was started on oral warfarin therapy for 3 to 6 months.

\subsection{Monitoring Indicators}

Patients were examined for calf tenderness and the Homans sign (gastrocnemius pain due to dorsiflexion) after surgery, and any lower extremity edema was noted. Laboratory testing was performed for D-dimer, and a concentration $>0.5 \mathrm{mg} / \mathrm{L}$ was defined as a positive finding. Lower extremity vascular Doppler ultrasound is the gold standard for the diagnosis of thrombosis [3].

\subsection{Statistical Methods}

The SPSS13.0 statistical software was used for statistical analysis. Qualitative data are expressed as percentages, rates were compared using the $\chi^{2}$ test, $P<0.05$ was the statistically significant threshold for comparisons between three groups, and $P<0.0125$ was the statistically significant threshold for pairwise comparisons.

\section{Results}

\subsection{Clinical Conditions of Patients in Three Groups after the Surgeries}

Some patients showed clinical symptoms of DVT, some displayed positive Homans sign without calf tenderness while others had calf tenderness but did not have a Homans sign. Only a few patients exhibited lower extremity edema. Calf tenderness was noted in 24 patients (14.7\%) in group 1, 15 patients (10.1\%) in group 2, and 20 patients $(8.5 \%)$ in group 3. Homans sign was found in 22 cases $(13.5 \%)$ in group 1,6 cases $(4.0 \%)$ in group 2, and 11 cases $(4.7 \%)$ in group 3. Lower extremity edema was present in 18 cases (11.0\%; group 1$), 3$ cases $(2.0 \%$; group 2), and 7 cases (2.9\%; group 3 ). These three clinical indicators were not highly specific and therefore were not used for statistical analysis. The D-dimer test was positive in 64 cases (39.3\%) in group 1, 38 cases (25.5\%) in group 2, and 62 cases (26.3\%) in group 3. The D-dimer detection threshold correlates with the occurrence of thrombosis but only serves as a secondary detection index. Lower extremity vascular Doppler ultrasound is the gold standard for the diagnosis of DVT and was performed by physicians who were associate chiefs or above 
in the ultrasound department of our hospital. All DVT cases were accompanied by the aforementioned clinical examination and a positive D-dimer test.

\subsection{Incidence of Thrombosis for Three Groups of Patients}

Through three adjustments to the clinical therapeutic regimen, the incidence of thrombosis declined to 15 cases $(9.2 \%)$ in group 1,3 cases $(2.0 \%)$ in group 2 , and 6 cases $(2.5 \%)$ in group 3 . A statistically significant difference was detected between group 1 (no low molecular weight heparin) and groups 2 and 3 (addition of low molecular weight heparin). However, the difference in incidence between groups 2 and 3, both receiving low molecular weight heparin, was not statistically significant (Table 1). During the post-operative follow-up period, the occurrence of thrombosis was 11/125 (8.8\%), 2/112 (1.8\%), and $3 / 202(1.5 \%)$ for groups 1,2 , and 3 , respectively. The differences between groups 1 and 2 and between groups 1 and 3 were found to be statistically significant, but the difference between groups 2 and 3 was not (Table 2).

\section{Discussion}

DTV is caused by abnormal coagulation of blood in the deep veins with perturbations in venous return due to blockage and is a common complication after major general surgery. The main causes of thrombosis include stagnant blood flow, vascular intimal injury, and hypercoagulable states. General surgeries are usually not associated with intimal injuries caused by limb contortions or lower limb vascular traction injuries. Instead, intimal injuries may be caused by prolonged surgery or bed rest in the elderly or those with significant postoperative pain, resulting in stagnant venous flow. Clinical preventative treatments include lower limb elevation, in-bed activities, lower limb muscle massage, direct current stimulation of lower limb muscle contraction and air pressure limb compression. Elderly patients often present with complications such as cardiovascular disease, diabetes, hypertension, hyperlipidemia, and other chronic diseases. In addition, surgery can induce hypercoagulable states and blood loss or blood transfusions during surgery are risk factors for thrombosis. Presently, intimal injuries caused by limb contortion and traction should be avoided, and a variety of therapeutic options are available to effectively treat stagnant venous flow. Assuming that no further advancements in surgical techniques and nursing skills will be made, altering blood hypercoagulability is critical. Although the evidence in the literature suggests several drugs for perioperative prevention of thrombosis, standardized prevention of deep vein thrombosis has not been implemented.

Beginning at the end of 2006, the gastrointestinal surgery center at our institution performed standardized preventative treatment of deep venous thrombosis for general surgery cases. After three clinical periods, we have identified the optimal duration and dosage for DVT prevention. Our statistical analyses have demonstrated that the thrombosis incidence of groups 2 and 3 is significantly lower than group 1 , indicating that the combined administration of three drugs is effective and crucial in the prevention of postoperative thrombosis. During the follow-up period, the thrombosis incidence of groups 2 and 3 remained lower than that of group 1, suggesting that this combination of drugs has long-term efficacy but requires further research.

As a universally-accepted efficacious anticoagulant, low molecular weight heparin does not increase the incidence

Table 1. Postoperative comparison of D-dimer and lower extremity vascular Doppler ultrasound between three groups of patients.

\begin{tabular}{cccc}
\hline Groups & Cases & D-dimer testing positive (\% of cases) & Lower extremity vascular Doppler ultrasound positive (\% of cases) \\
\hline Group 1 & 163 & $39.3(64 / 163)$ & $9.2(15 / 163)$ \\
Group 2 & 149 & $25.5(38 / 149)$ & $2.0(3 / 149)$ \\
Group 3 & 236 & $26.3(62 / 236)$ & $2.5(6 / 236)$ \\
Statistical Value & & 9.67 & 12.95 \\
$P$-Value & & 0.008 & 0.002 \\
\hline
\end{tabular}

Table 2. Follow-up comparison of D-dimer and lower extremity vascular Doppler ultrasound of the three groups of patients.

\begin{tabular}{cccc}
\hline Groups & Cases & D-dimer testing positive (\% of cases) & Lower extremity vascular Doppler ultrasound positive (\% of cases) \\
\hline Group 1 & 125 & $30.4(38 / 125)$ & $8.8(11 / 125)$ \\
Group 2 & 112 & $16.1(18 / 112)$ & $1.8(2 / 112)$ \\
Group 3 & 202 & $17.8(36 / 202)$ & $1.5(3 / 202)$ \\
Statistical Value & & 10.64. & 13.25 \\
$P$-Value & & 0.010 & 0.001 \\
\hline
\end{tabular}


of abdominal bleeding [4,5]. In our study, we did not observe abdominal bleeding even in second-surgery cases treated with low molecular weight heparin. Postoperative bleeding is mainly influenced by surgical technique and hemostasis during surgery. However, due to a lack of stable anticoagulant antagonistic drugs, high dosage of low molecular weight heparin is not recommended. Presently, patients that undergo major abdominal surgeries at our hospital are no longer routinely treated with hemostatic drugs if the pre-surgical liver function and coagulation tests are normal and intraoperative hemostasis is complete. For the preventative treatment of thromboses, we implemented the following clinical progression: from January 2007 to March 2008, we only used dextran and IV salvia for thrombosis prevention with the goal to inhibit platelet aggregation and decrease blood viscosity. In April 2008, no more than 40 mg of intradermal low molecular weight heparin was added daily as the standard preventative dose. Theoretically, the preventative efficacy of low molecular weight heparin for thrombosis should improve with early administration. However, our study found that the difference in thrombosis incidence between administration of low molecular weight heparin on the first (group 2) or third postoperative day (group 3) is not statistically significant. In addition, after one year of clinical observations, we found that the administration of low molecular weight heparin on the day of the surgery increases the occurrence of postoperative wound exudate (drainage fluid), peritoneal effusion, and intractable fever (specific cases are described in the supplementary information). Therefore, since April 2009, we have postponed the administration of low molecular weight heparin to postoperative day 3 and, until April of 2010, the clinically observed adverse symptoms have been significantly reduced.

\section{Conclusion}

Previous reports in the literature have recommended the administration of low molecular weight heparin for up to 28 days in postoperative abdominal tumor patients [6]. Additional evidence indicates that the standard prophylactic dose of low molecular weight heparin may lead to the decreased endogenous anti-Xa factor level, hence increasing the incidence of DVT [7]. In this retrospective clinical study, we used a triad of drugs for the prevention of thrombosis and clinical groups were divided based on the clinical response. Our understanding of postoperative anticoagulation therapy was gradually improved. During the follow-up period of this study, randomized control trials can lead to results that may be more convincing. We can also expand the sample size, adjust the dosage, and critically analyze the starting point of low molecular weight heparin or the intergroup differences in the duration of drug use. We hope to study the drugs for the prevention of thrombosis in more depth and minimize the adverse effects. In conclusion, we believe the most effective therapy that minimizes side effects for abdominal tumor surgeries postoperatively is a regimen that includes the initiation of a 7-day course of low molecular weight dextran and IV salvia immediately after surgery with the addition of a 7-day course of low molecular weight heparin three days after surgery.

\section{REFERENCES}

[1] J. A. Heit, D. N. Mohr, M. D. Silverstein, et al., "Predictors of Recurrence after Deep Vein Thrombosis and Pulmonary Embolism: A Population-Based Cohort Study," Archives of Internal Medicine, Vol. 160, No. 6, 2000, pp. 761-768. http://dx.doi.org/10.1001/archinte.160.6.761

[2] H. Y. Cheung, C. C. Chung, K. K. Yau, et al., "Risk of Deep Vein Thrombosis Following Laparoscopic Rectosigmoid Cancer Resection in Chinese Patients," Asian Journal of Surgery, Vol. 31, No. 2, 2008, pp. 63-68. http://dx.doi.org/10.1016/S1015-9584(08)60060-3

[3] R. N. Beck, A. Kuzmin, D. Koren, et al., "Asymptomatic Deep Vein Thrombosis in Advanced Cancer Patients: The Value of Venous Sonography,” Journal of Clinical Ultrasound, Vol. 38, No. 5, 2010, pp. 232-237.

[4] S. Schulman, "Low Molecular Weight Heparin-A Safer Anticoagulant in Thrombosis and Cancer," Läkartidningen, Vol. 101, 2004, pp. 1712-1715.

[5] M. Levine, M. Gent, J. Hirsh, et al., "A Comparison of Low-Molecular-Weight Heparin Administered Primarily at Home with Unfractionated Heparin Administered in the Hospital for Proximal Deep-Vein Thrombosis," The New England Journal of Medicine, Vol. 334, 1996, pp. 677-681. http://dx.doi.org/10.1056/NEJM199603143341101

[6] J. Muntz, "Duration of Deep Vein Thrombosis Prophylaxis in the Surgical Patient and Its Relation to Quality Issues,” The American Journal of Surgery, Vol. 200, No. 3, 2010, pp. 413-421. http://dx.doi.org/10.1016/j.amjsurg.2009.05.045

[7] D. Malinoski, F. Jafari, T. Ewing, et al., "Standard Prophylactic Enoxaparin Dosing Leads to Inadequate Anti-Xa Levels and Increased Deep Venous Thrombosis Rates in Critically Ill Trauma and Surgical Patients," Journal of Trauma, Vol. 68, No. 4, 2010, pp. 874-880. 